\title{
EXPERIMENTAL SUBSTANTIATION OF THE COMPOSITION OF THE GEL BASE WITH HYDROCORTISONE FOR USE IN VETERINARY MEDICINE
}

\author{
Viktoriia Pul-Luzan, Olga Rukhmakova
}

As veterinary practice shows, most often atopic dermatitis in animals manifests itself in the form of a rash (in the ears, muzzle, paws, etc.), which in turn is accompanied by itching. First of all, prescribe a single injection of glucocorticoids, or short-term therapy. Corticosteroid hormones (glucocorticoids) are one of the most powerful antiallergic drugs. They are effective in treating almost all types of allergic reactions. An appropriate dose of glucocorticoids is required to obtain a rapid effect, and the form of the drug should be convenient to use.

The aim of the research. The aim of our study was experimentally substantiate the choice of the optimal gelling agent when developing a gel composition with hydrocortisone for use in veterinary medicine.

Materials and methods. Physical, physico-chemical and pharmaco-technological methods were used during the experimental study. Studies were performed according to the method described in the SPhU. The rheological properties of the samples were determined using a rotary viscometer type Brookfield HB DV (USA) with spindle SC4-21.

Results. As a result of the research, it was found that Aristoflex as a gelling agent in the development of a veterinary drug of local action will ensure the availability of appropriate extrusion properties (namely, easy and uniform application of animal skin, ease of use). Gels with hydrocortisone based on Aristoflex gave stable performance, which was confirmed by the results of mechanical and colloidal stability.

Conclusions. The composition and technology of a hydrocortisone gel for local therapy of atopic dermatitis in animals has been developed. Aristoflex at a concentration of $1.5 \%$ on the basis of a set of physicochemical, structuralmechanical and biopharmaceutical studies was selected as the optimal gelling agent in the drug

Keywords: gelling agent, Aristoflex, hydrocortisone, composition, technology, rheological studies, atopic dermatitis, veterinary

How to cite:

Pul-Luzan, V., Rukhmakova, O. (2021). Experimental substantiation of the composition of the gel base with hydrocortisone for use in veterinary medicine. ScienceRise: Pharmaceutical Science, 3 (31), 4-10. doi: http://doi.org/10.15587/2519-4852.2021.233664

\section{Introduction}

One of the most common diseases among animals is atopic dermatitis. According to statistics, compared to previous years, the number of animals affected by this pathology is rapidly increasing. The entire fault, according to experts, modern environmental conditions that provoke the susceptibility of animals to allergens.

Atopic dermatitis - a common disease of cats and dogs, described as a genetically caused itchy inflammation of the skin associated with the formation of $\operatorname{IgE}$ antibodies, usually directed against environmental allergens or airborne allergens. According to international statistics, 3 to $30 \%$ of dogs worldwide have atopic dermatitis [1].

Atopic dermatitis affects young animals aged 1 to 5 years, but can be diagnosed earlier. The first symptoms of dermatitis appear at the age of six months, when the allergen is introduced into the body and forces the immune system to produce antibodies-neutralizers, and then the pathology recurs throughout life.

Atopic dermatitis has a pronounced clinical picture. The symptoms include the main and additional symptoms. The main symptoms are: severe itching of the skin, which causes the animal great discomfort; skin damage, scratches, abrasions (especially in the face and paws), which appear due to the fact that the animal is constantly itching and tearing the skin with its claws; got into the wound infection provokes boils, hyperpigmentation, abscesses; hair loss, alopecia; characteristic odour from the ears, reminiscent of playing yeast dough $[2,3]$.

Additional signs of atopic dermatitis include excessive dryness of the skin; immediate reaction to the allergen; external form of allergic otitis; superficial manifestations of staphylococcal infection.

Symptomatic treatment is carried out at the initial stage of immunotherapy, but can be prescribed after: if the effect is short-lived or not fully manifested. Medications of different groups for the treatment of atopic dermatitis, as well as special shampoos for skin care are prescribed by a veterinarian, self-medication is dangerous to the health of the dog and can only worsen the situation [4].

It is necessary to prescribe corticosteroids. They have a powerful and rapid effect, reduce the activity of a number of inflammatory factors. These drugs are hormonal, their action is aimed at eliminating itching, allergic edema, redness. Corticosteroid hormones (glucocorti- 
coids) are one of the most powerful antiallergic drugs. They are effective in treating almost all types of allergic reactions. The reason is in their structural similarity to some adrenal hormones. The mechanism of their antiallergic action is very diverse: they reduce the permeability of capillaries, inhibit the inflammatory reaction and the growth of connective tissue, have the effect of replacement therapy in adrenal insufficiency. It has been established that glucocorticoids affect the development of any stage of allergic reactions, but most of all - the pathophysiological stage. At the same time, their antiinflammatory effect is maximally manifested [5].

Prednisolone, methylprednisolone, dexamethasone and hydrocortisone are most commonly prescribed [6, 7].

Pharmacodynamic effects of corticosteroids are realized due to their anti-inflammatory (including antiexudative and antiproliferative effects), immunosuppressive and antiallergic effects. These drugs also actively affect various metabolic processes in the body.

The most important pharmacological effect of corticosteroids in terms of pathogenesis of nephropathy is, in particular, suppression of edema and proliferation of resident glomerular cells (resulting in partial or complete obliteration of the vessels of the primary network of kidneys). They also reducing the degree of infiltration of the renal parenchyma, hence, irreversible processes of sclerosis in the renal parenchyma are reduced $[8,9]$.

Thus, the aim of our study was to create new veterinary drugs with hydrocortisone for local therapy of atopic dermatitis.

\section{Planning (methodology) of research}

When developing a soft veterinary drug, it is necessary to pay attention to the type of carrier used, nature and physicochemical properties of active pharmaceutical ingredients, excipients, take into account the influence of $\mathrm{pH}$, temperature and other factors on structural and mechanical, technological properties of the developed gel base. These factors contribute to the release and absorption of active pharmaceutical ingredients from the mild dosage form. To establish a rational gelling agent, we used the following gelling agents: Carbopol Ultrez 21, Sepimax and Aristoflex. Selected gel repellents are approved for use in veterinary pharmacy.

The concentration of hydrocortisone for topical therapy (1\%) was selected based on analysis of literature sources and propylene glycol (10\%) because of studies. Model samples were prepared by the technology of gel preparation generally prescribed in the SPhU.

To establish the optimal gelling agent, rheological studies of the developed samples of extemporaneous gels with hydrocortisone were performed.

\section{Material and methods}

Modern physical, physicochemical and pharmacotechnological methods were used during the experimental study. The determination of homogeneity was performed according to the method described in the SPhU I ed., p. 511. Take five samples of each sample of 20-30 mg each, place two samples on a glass slide, cover with a second glass slide and press firmly to form spots with a diameter of about $2 \mathrm{~cm}$.
As active and additional components were used:

- Carbopol Ultrez 21 - USA, manufacturer Lubrizol;

- triethanolamine (Triethanolamine) - Ukraine;

- Sepimax Zen - France, manufacturer Seppic;

- Aristoflex (AVC) - Switzerland;

- propylene glycol (Propylene glycol) - Germany;

- hydrocortisone acetate - suspension for injection $25 \mathrm{mg} / \mathrm{ml}, 2 \mathrm{ml}$, JSC "Farmak".

The obtained samples were examined with the naked eye (at a distance of about $30 \mathrm{~cm}$ from the eyes). The sample was considered homogeneous if no visible particles, foreign inclusions and signs of physical instability were detected in all four samples. If one of the samples did not pass the test, the determination was performed on an additional eight samples, and all eight samples had to pass the test.

Determination of thermal stability was carried out according to methods, which are presented in the National Standard of Ukraine "Cosmetic creams. General technical conditions: DSTU 4765:2007”.

A tube of $8.0 \pm 2.0 \mathrm{~g}$ of test samples was placed in a thermostat with a temperature of $(40 \pm 2){ }^{\circ} \mathrm{C}$ was left for 1 week, then placed in a refrigerator with the appropriate temperature $(10 \pm 2){ }^{\circ} \mathrm{C}$ for 1 week, then kept for 3 days at room temperature $\left(15-25^{\circ} \mathrm{C}\right)$. The stability of the studied gel samples was determined visually - in the absence of delamination.

Determination of colloidal stability. The tubes were filled with test gel samples by $2 / 3$ of the volume (approximately $9.0 \mathrm{~g}$ ) of the test samples (so that the masses of the test tubes with the drug did not differ by more than $0.02 \mathrm{~g}$ ), and weighed to the nearest $0.01 \mathrm{~g}$. The tubes were then placed in a water bath at the desired temperature $(42.5 \pm 2.5){ }^{\circ} \mathrm{C}$ for $20 \mathrm{~min}$, then wiped dry on the outside and placed in centrifuge wells. Centrifuged for $5 \mathrm{~min}$ at a speed of $6000 \mathrm{rpm}$. The stability of the studied gel samples was determined visually - in the absence of delamination.

The rheological properties of the samples were determined using a rotary viscometer type Brookfield HB DV (USA), spindle SC4-21.

Thermostating of the samples was performed using a thermostat. A portion of the gel of about $(17.0 \pm 0.5) \mathrm{g}$ was placed in the container of the outer stationary cylinder. The required experiment temperature was set using a thermostat, then the required experiment conditions were set using software (shear rate gradient, number of experiment points on the sample flow curve and measurement duration at each point of the curve). The device allows to measure the shear stress in the range of 0.5-3.0 104 PA, shear rate from 0.1 to $4000 \mathrm{~s}^{-1}$, viscosity $-1-109 \mathrm{mPa} x \mathrm{~s}$.

For a more complete study of gel samples, the indicators of their mechanical stability (MS) were calculated. It is known that the optimal value of MS is 1 . The value of MS is defined as the ratio of the value of the tensile strength of the structure to failure $\left(\tau_{1}\right)$ to the value of the tensile strength after failure $\left(\tau_{2}\right)$.

To study the extrusion properties according to the indicators of rheological studies calculated the coefficients of dynamic vacuum $(\mathrm{Kd})$ for the drug during the shelf life.

Statistical processing of the results of pharmacotechnological, physicochemical, biopharmaceutical stud- 
ies was performed in accordance with the methods of SPU 2.3, paragraph 5.3.

The bioavailability (release) of hydrocortisone acetate was studied by dialysis through a semi-permeable membrane by Hoechst in vitro experiments. The study was performed at a temperature of $(37.0 \pm 0.5){ }^{\circ} \mathrm{C}$. The dialysis medium was water $R$. Thermostating was performed in an ultrathermostat TC-16A (Germany) with an accuracy of $\pm 0.1{ }^{\circ} \mathrm{C}$. The temperature was measured with a laboratory thermometer. Quantitative determination of hydrocortisone acetate in the dialysate was carried out by HPLC using a mobile phase of methanol - water 70:30 according to the proposed method. Sample weight for experiment -2.0 .

Test solution. A weighed portion of the preparation corresponding to $5.0 \mathrm{mg}$ of hydrocortisone acetate is placed in a separating funnel with a capacity of $100 \mathrm{ml}$, $50 \mathrm{ml}$ of isooctane is added and shaken until the gel dissolves. Add $15 \mathrm{ml}$ of $80 \%$ methanol and shake for 30 minutes. After stratification of liquids, the lower layer is placed in a volumetric flask with a capacity of $50 \mathrm{ml}$, the extraction is repeated twice, each time using $15 \mathrm{ml}$ of $80 \%$ methanol, and the extracts are combined. Bring the volume of the resulting solution to the mark with $80 \%$ methanol and filter. $1.0 \mathrm{ml}$ of the resulting solution is placed in a $25 \mathrm{ml}$ volumetric flask and the volume of the solution is brought to the mark with methanol.

Standard sample solution. About $10.0 \mathrm{mg}$ (accurately weighed) of a standard sample of hydrocortisone acetate is placed in a volumetric flask with a capacity of $50 \mathrm{ml}$, dissolved in $30 \mathrm{ml}$ of methanol and the volume of the solution is brought to the mark with the same solvent. $1.0 \mathrm{ml}$ of the resulting solution is placed in a volumetric flask with a capacity of $50 \mathrm{ml}$ and the volume of the solution is brought to the mark with methanol.

Chromatographic conditions:

Column: $25 \times 0.46 \mathrm{~cm}$, silica gel for chromatography $(\mathrm{C} 18), 10 \mu \mathrm{m}$;

Flow rate: $1.0 \mathrm{ml} / \mathrm{min}$;

Detector: spectrophotometric, $240 \mathrm{~nm}$;

Sample's volume: $20 \mu \mathrm{l}$;

The chromatography time is 2 times the retention time of the main peak.

The content of hydrocortisone acetate as a percentage of the declared amount $(\mathrm{X})$ is calculated by the formula:

$$
X=\frac{S_{1} \cdot a_{0} \cdot 1 \cdot P \cdot 50 \cdot 25}{S_{0} \cdot 50 \cdot 50 \cdot a_{1} \cdot 1 \cdot L}=\frac{S_{1} \cdot a_{0} \cdot P}{S_{0} \cdot a_{1} \cdot L \cdot 2},
$$

where $S_{1}$ is the peak area of hydrocortisone acetate on the chromatogram of the test solution;

$\mathrm{S}_{0}$ is the area of the hydrocortisone acetate peak in the chromatogram of the standard sample solution;

$a_{1}$ is the weight of a sample of the drug, mg;

$\mathrm{a}_{0}$ is the weight of a standard sample of hydrocortisone acetate, $\mathrm{mg}$;

$\mathrm{P}$ is the content of hydrocortisone acetate in a standard sample of hydrocortisone acetate, \%;

$\mathrm{L}$ is the stated amount of hydrocortisone acetate in the drug, g/g.

\section{Results}

The composition of the veterinary medicinal product for the topical treatment of atopic dermatitis should include an active pharmaceutical ingredient that has targeted anti-inflammatory, antimicrobial and reparative effects. In addition, it should have the appropriate properties to improve the condition of animal skin $[8,9]$. Hydrocortisone - a hormone secreted by the adrenal cortex - glucocorticoid. Pharmacological action of hydrocortisone - anti-inflammatory, antiallergic, immunosuppressive, antipruritic, antishock, antiexudative, glucocorticoid. When applied to the skin: inflammatory and allergic skin diseases of non-microbial etiology, including eczema, dermatitis (allergic, atopic, bullous herpetiform, exfoliative, seborrheic, contact); itchy dermatoses, photodermatoses, anogenital itching, insect bites, pemphigus, erythroderma, psoriasis $[10,11]$.

Given the above, the gel for the treatment of atopic dermatitis in animals as active component was chosen glucocorticoid - hydrocortisone at a concentration of $1 \%$ [12].

To install a rational gelling agent, we have prepared the following model samples. The concentration of hydrocortisone $(1 \%)$ was selected based on analysis of literature sources and propylene glycol $(10 \%)$ based on the results of preliminary studies of osmotic activity of model samples of the gel (Table 1).

The results of the obtained data are presented in Table 2.

Table 1

Composition of model samples of gels with hydrocortisone acetate

\begin{tabular}{|c|l|c|c|c|}
\hline \multirow{2}{*}{ No. Active and excipients } & \multicolumn{1}{|c|}{ Samples } \\
\cline { 3 - 5 } & & No. 1 & No. 2 & No. 3 \\
\hline 1. & Carbopol Ultrez 21 & 1.5 & - & - \\
\hline 2. & Trometamol & 1.5 & - & - \\
\hline 3. & Sepimax & - & 1.5 & - \\
\hline 4. & Aristoflex & & & 1.5 \\
\hline 5. & Hydrocortisone & 1.0 & 1.0 & 1.0 \\
\hline 6. & Propylene glycol & 10.0 & 10.0 & 10.0 \\
\hline 7. & Purified water & & Up to 100.0 \\
\hline
\end{tabular}


The results of the study of the physicochemical and pharmaco-technological properties of the studied gel samples

\begin{tabular}{|c|c|c|c|c|}
\hline \multirow{2}{*}{ No. } & \multirow{2}{*}{ Indexes } & \multicolumn{3}{|c|}{ Samples } \\
\hline & & No. 1 & No. 2 & No. 3 \\
\hline 1. & Appearance & $\begin{array}{l}\text { Gel transparent white } \\
\text { color }\end{array}$ & $\begin{array}{l}\text { Gel transparent white } \\
\text { color }\end{array}$ & $\begin{array}{l}\text { Gel transparent white } \\
\text { color }\end{array}$ \\
\hline 2. & $\mathrm{pH}$ of $10 \%$ solution & $7.6 \pm 0.2$ & $7.5 \pm 0.3$ & $5.5 \pm 0.2$ \\
\hline 3. & Structural viscosity, $\eta(\mathrm{mPa} \cdot \mathrm{s})$ & 6800 & 1900 & 3700 \\
\hline 4. & Colloidal stability, (at $6000 \mathrm{rpm}$ ) & delamination & delamination & stable \\
\hline 5. & Mechanical stability (MS) & 1.63 & 1.55 & 1.33 \\
\hline
\end{tabular}

According to experimental data, we excluded from further studies samples No. 1, 2. In the studied samples at once after their preparation during the study of colloidal stability under the influence of a complex of active substances, the gel structure was destroyed (delamination in the tubes was observed).

Thus, for further research we selected a sample No. 3 based on Aristoflex at a concentration of $1.5 \%$, which had satisfactory consumer, physicochemical and structural-mechanical properties, respectively.

The next stage of our research was to study the effect of selected substances on the rheoparameters of the gel system with Aristoflex:

- sample A - gel base;

- sample B - gel base + hydrocortisone; ene glycol

- sample C - gel base + hydrocortisone + propyldrocortisone

- sample D - gel base + propylene glycol + hy-

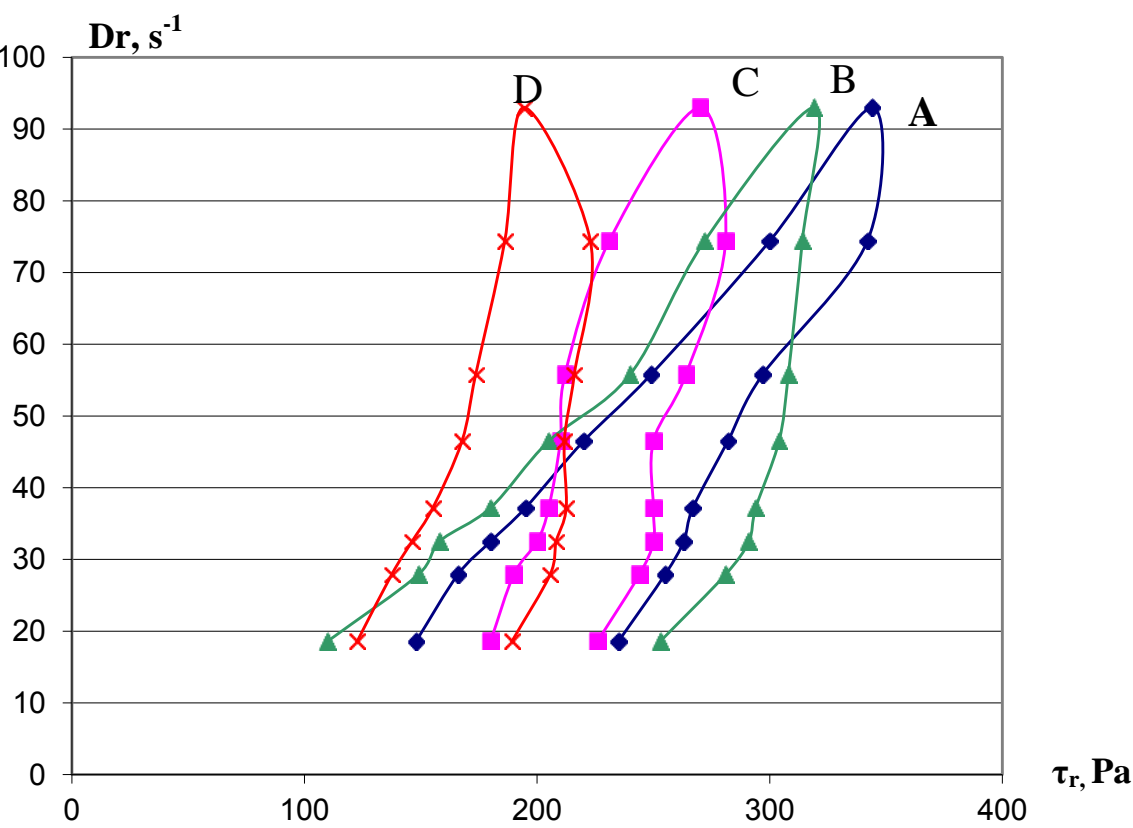

Fig. 1. Rheograms of gels: A - gel base; B - gel base + hydrocortisone;

$\mathrm{C}-$ gel base + hydrocortisone + propylene glycol; D - gel base + propylene glycol + hydrocortisone
The obtained rheograms (Fig. 1) show that with the introduction of active (hydrocortisone) and auxiliary (polyethylene glycol) the type of flow did not change and was characterized as plastic.

The results of our research show that the structural viscosity was restored when the shear stress was reduced. The "ascending" curves of the hysteresis loops indicated a decrease in structural viscosity after destruction of the structure of hydrocortisone gels, and the "descending" curves reflected the optimal state of equilibrium in which the systems were after destruction. It is noted that the addition of hydrocortisone reduced the rheoparameters, and it was proved that the technology of introduction of sample $\mathrm{C}$ is rational: first hydrocortisone, then propylene glycol. It is his system that is more structured, which is primarily due to the addition of glucocorticosteroids.

These data are confirmed in digital format and are shown in Table 3. 
From the data presented in the Tab Table 3 it can be concluded that the two samples have satisfactory performance as for a stable gel system. However, for further research, we selected the sample $\mathrm{C}$, which has higher viscosity and better values of mechanical stability (MS), namely 13100 and 1.16 , respectively.

When studying the dependence of the structural viscosity on the shear rate gradient, it is seen that the structural viscosity of the studied gel samples gradually decreased with increasing shear rate gradient (Fig. 2). This dependence is also characteristic of systems that have a plastic type of flow and characterizes the studied samples of gels as structured dispersed systems, in which the addition of active and excipients does not interact with the developed gel base, and provides uniform and gradual application to the skin.

When studying the release of API (hydrocortisone acetate) from the studied model samples, it was found that the most complete release of hydrocortisone acetate provides sample C. Excipients in its composition provide a prolonged release of hydrocortisone acetate at the level of $5-6 \mu \mathrm{g} / \mathrm{ml}$ in the study form).

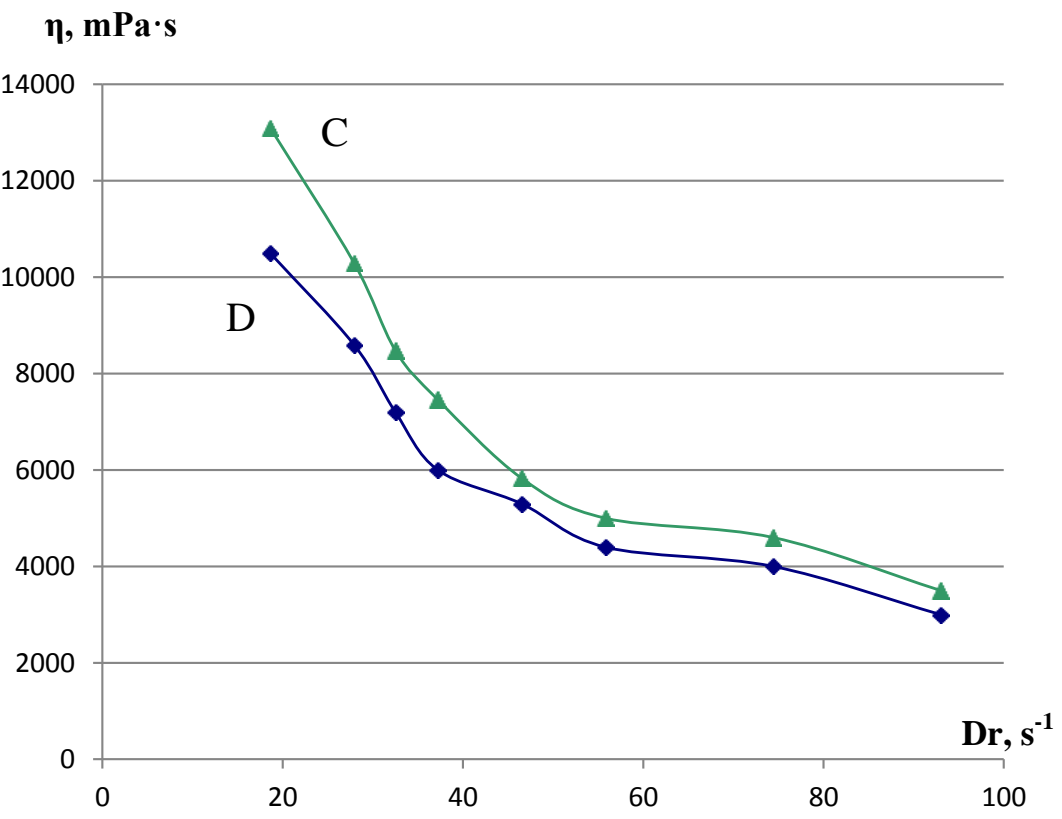

Fig. 2. Dependence of structural viscosity of gels with hydrocortisone on shear rate: sample C, sample D

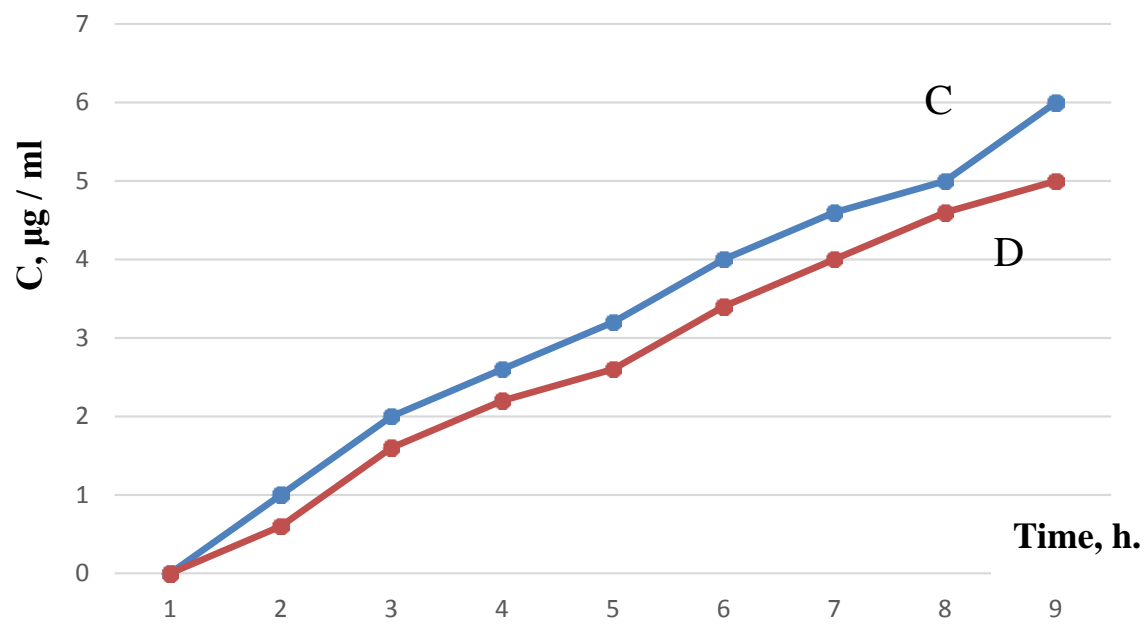

Fig. 3. Kinetics of hydrocortisone acetate release from model gels based on sample C and sample D

Based on the results of the studies, which are shown in the diagram (Fig. 3), it is seen that the release of the active ingredient (hydrocortisone) from samples $\mathrm{C}$ and $\mathrm{D}$ is almost the same. However, taking into account previous studies, namely the indicators of mechanical stability (MS), we chose sample C based on Aristoflex for further studies.

\section{Discussion}

The disease atopic dermatitis in dogs is a common disease. Scientists around the world have performed a series of laboratory card analyzes in veterinary clinics for the treatment of this disease. A number of studies have proven that glucocorticosteroids are highly effective in the treatment of this disease $[12,13]$. 
For the treatment of this pathology, we have chosen a topical preparation in the form of a gel with glucocorticosteroids. As you know, there are glucocorticosteroids that are used only internally or only externally. That is why our choice is hydrocortisone, which has strong antiinflammatory and antipruritic effects precisely locally. Foreign studies confirm the effectiveness of topical application of this glucorticosteroid when used topically $[14,15]$.

For the experiment, we prepared samples of experimental gels with hydrocortisone with the selected gelling agents. Three samples of the gel were investigated with hydrocortisone (1\%) on different gel wasps, namely Carbopol, Sepimax and Aristophlex. Based on the studies carried out (appearance, $\mathrm{pH}$, structural viscosity, colloidal and mechanical stability), it was found that the sample based on Aristolex had positive results and had colloidal stability, in contrast to the samples based on Carbopol and Sepimax [15, 16]. According to the results of the studies, the samples based on Carpobol and Sepimax were destroyed by the gel structure (color change of the gel, the presence of impurities). Therefore, for further research, a sample based on Aristoflex was selected, which gave satisfactory consumer properties and was stable during storage.

Thus, we can conclude that the use of Aristoflex as a gelling agent in the development of a veterinary drug of local action will ensure the availability of appropriate extrusion properties (namely, easy and uniform application of animal skin, ease of use). To confirm the rational technology of introducing the active components of the gel, rheological studies were carried out.

Study limitation. The studies conducted on the rationale for the choice of a gelling agent when creating a stable extemporal gel with hydrocortisone do not fully reflect the possible risks of obtaining this dosage form prepared in a pharmacy. The results obtained from the studies performed require supporting studies when performing the validation of this process.

Prospects for future research. During the experiment, the selected concentrations of gelling agents were used in a single concentration, namely $1.5 \%$, although the range of use of these gelling agents was from $1 \%$ to $5 \%$. The purpose of further research: to carry out a number of experiments with different concentrations of the selected gelling agents and to conduct biopharmaceutical studies on the rate of release of the active component from the dosage form.

\section{Conclusions}

The structural and mechanical parameters of experimental samples of extemporaneous gel with hydrocortisone for use in veterinary medicine were studied. To establish a rational gelling agent, we prepared samples based on Carbopol ultras 21, Sepimax and Aristoflex. According to the literature, the concentration of hydrocortisone when applied topically. Model samples were prepared by the technology of gel preparation generally prescribed in the SPU. With the help of pharmacotechnological and rheological studies, it was found that the use of Aristoflex as a gelling agent in the development of a topical veterinary drug with hydrocortisone would ensure the availability of appropriate extrusion properties (namely easy and even application of animal skin, ease of use).

\section{Conflict of interests}

The authors declare that they have no conflicts of interest.

Financing

None

\section{References}

1. Bajwa, J. (2016). Atopic dermatitis in cats. The Canadian Veterinary Journal, 59 (3), 311-313.

2. Hensel, P., Santoro, D., Favrot, C., Hill, P., Griffin, C. (2015) Canine atopic dermatitis: detailed guidelines for diagnosis and allergen identification. BMC Veterinary Research. 11 (196), 46-54. doi: http://doi.org/10.1186/s12917-015-0515-5

3. Marsella, R., Segarra, S., Ahrens, K., Alonso, C., Ferrer, L. (2020). Topical treatment with SPHINGOLIPIDS and GLYCOSAMINOGLYCANS for canine atopic dermatitis. BMC Veterinary Research, 16 (1). doi: http://doi.org/10.1186/s12917-020-02306-6

4. Santoro, D. (2019). Therapies in Canine Atopic Dermatitis: An Update. Veterinary Clinics of North America: Small Animal Practice, 49 (1), 9-26. doi: http://doi.org/10.1016/j.cvsm.2018.08.002

5. Fridman-Bengtsson, O., Höybye, C., Porthén, L., Stjärne, P., Hulting, A.-L., Sunnergren, O. (2019). Evaluation of different hydrocortisone treatment strategies in transsphenoidal pituitary surgery. Acta Neurochirurgica, 161 (8), 1715-1721. doi: http://doi.org/10.1007/ s00701-019-03885-6

6. Mueller, R. S. (2019). Update on Allergen Immunotherapy. Veterinary Clinics of North America: Small Animal Practice, 49 (1), 1-7. doi: http://doi.org/10.1016/j.cvsm.2018.08.001

7. Ibrahim, F., El-Deen, A. K., Shimizu, K. (2018). Comparative study of two different chromatographic approaches for quantitation of hydrocortisone acetate and pramoxine hydrochloride in presence of their impurities. Journal of Food and Drug Analysis, 26 (3), 1160-1170. doi: http://doi.org/10.1016/j.jfda.2017.12.008

8. Folster-Holst, R., Abeck, D., Torrelo, A. (2016). Topical hydrocortisone 17-butyrate 21-propionate in the treatment of inflammatory skin diseases: pharmacological data, clinical efficacy, safety and calculation of the therapeutic index. Pharmazie, 71 (3), $115-121$.

9. Kligman, A. M., Kaidbey, K. H. (1978). Hydrocortisone revisited. An historical and experimental evaluation. Cuties, 22 (2), 232-244.

10. Bruet, V., Bourdeau, P. J., Roussel, A., Imparato, L., Desfontis, J.-C. (2012). Characterization of pruritus in canine atopic dermatitis, flea bite hypersensitivity and flea infestation and its role in diagnosis. Veterinary Dermatology, 23 (6), 487-493. doi: http://doi.org/10.1111/ j.1365-3164.2012.01092.x

11. Favrot, C., Steffan, J., Seewald, W., Picco, F. (2010). A prospective study on the clinical features of chronic canine atopic dermatitis and its diagnosis. Veterinary Dermatology, 21 (1), 23-31. doi: http://doi.org/10.1111/j.1365-3164.2009.00758.x

12. Miller, W. H., Griffin, C. E. (2013). Small animal dermatology. St. Louis: W.B. Elsevier, 24 (3), 57-107. 
13. Olivry, T., DeBoer, D. J., Prélaud, P., Bensignor, E. (2007). Food for thought: pondering the relationship between canine atopic dermatitis and cutaneous adverse food reactions. Veterinary Dermatology, 18 (6), 390-391. doi: http://doi.org/10.1111/j.13653164.2007.00625.x

14. Lourenço, A. M., Schmidt, V., São Braz, B., Nóbrega, D., Nunes, T., Duarte- Correia, J. H. et. al. (2016). Efficacy of proactive long-term maintenance therapy of canine atopic dermatitis with $0.0584 \%$ hydrocortisone aceponate spray: a double-blind placebo controlled pilot study. Veterinary Dermatology, 27 (2), 88. doi: http://doi.org/10.1111/vde.12285

15. Yarnykh, T. G., Tykhonov, O. I., Melnyk, G. M., Yuryeva, G. B. (2017). Pharmacopoeian aspects of suspensions preparation in pharmacy conditions. Asian Journal of Pharmaceutics, 11 (4), 859-864.

16. Rukhmakova, O. A., Yarnykh, T. G., Pul-Luzan, V. V., Kotenko, O. M., Buryak, M. V. (2020). Experimental research on the development of extemporaneous ointment for the treatment of proctologic diseases. Journal of Global Pharma technology, 12 (1), $229-234$.

Received date 25.02.2021

Accepted date 03.06.2021

Published date 30.06.2021

Viktoriia Pul-Luzan*, PhD, Assistant, Department of Drugs Technology, National University of Pharmacy, Pushkinska str., 53, Kharkiv, Ukraine, 61002

Olga Rukhmakova, Doctor of Pharmaceutical Sciences, Professor, Department of Drugs Technology, National University of Pharmacy, Pushkinska str., 53, Kharkiv, Ukraine, 61002

*Corresponding author Viktoriia Pul-Luzan, e-mail: pulluzanv@gmail.com 